\title{
Patterns of Motoneuron Dysfunction and Recovery
}

\author{
ALAN MCCOMAS, ADRIAN UPTON AND PER JORGENSEN
}

SUMMARY: Electrophysiological studies have been carried out on five patients with neuropathies of different etiologies. In each patient serial estimates were made of the numbers of functioning motor units in various muscles. It was found that the intensity of the neuropathic process and the rate of recovery differed in a consistent way among the motoneuron pools investigated. The lesion was more severe in extensor digitorum brevis neurons than in thenar neurons, while the hypothenar ones were least affected. A stage of partial synaptic failure has been recognized in

RÉSUMÉ: Des études électrophysiologiques ont été entreprises sur 5 patients atteints de neuropathies de différentes étiologies. Sur chaque patient, une série d'estimations ont été faites sur le nombre d'unités motrices fonctionnelles de muscles divers. Il a été trouvé que l'intensité du procédé neuropathique et que le taux de recouvrement différaient de façon constante parmi les groupes de motoneurones investigués. La lésion était plus sévère dans les neurones des courts extenseurs des doigts que dans les neurones de l'éminence thénar, cependant que les neurones des éminences hypothénars étaient les moins atteints. Une phase de déficience synaptique partielle a été remarquée pendant which a motoneuron appears to be no longer able to excite a muscle fiber, but still capable of maintaining certain trophic activities. By comparing the number of functioning motor units with the size of the maximum evoked muscle response it has been possible to detect the adoption of denervated muscle fibers by axonal sprouts from 'healthy' surviving neurons (collateral reinnervation). Lastly, in some muscles it appears that the adopted muscle fibers may subsequently be recaptured by the original motoneurons following recovery of the latter from the neurotoxic insult.

laquelle un motoneurone ne semble plus être en mesure d'exciter une fibre musculaire, quoique toujours capable de maintenir certaines activités trophiques. En comparant le nombre d'unité motrices fonctionnelles avec l'intensité du maximum de réponse du muscle, il a été possible de détecter la jonction ("adoption") de fibres musculaires dénervées à des bourgeons axonaux provenant des neurones "sains" survivants (réinnervation collatérale). Il apparait en dernier lieu, que dans quelques muscles les fibres musculaires "adoptées' peuvent être subséquemment "recaptées' par les motoneurones originaux, lorsque ces derniers récupèrent de l'insulte neurotoxique.
From the Medical Research Council's Developmental Neurobiology Research Group and the Department of Medicine, McMaster University Medical Center, 1200 Main St. W., Hamilton, Ontario.

Reprint requests to Dr. Alan McComas, McMaster University Medical Center, 1200 Main St. W., Hamilton, Ontario.
In 1971, McComas, Fawcett, Campbell and Sica described a simple electrophysiological method for obtaining rough estimates of the numbers and sizes of functioning motor units in the human extensor digitorum brevis (EDB) muscle. The technique was then applied, with some modifications, to the study of thenar muscles by Brown (1972) while McComas and colleagues adapted it to the thenar, hypothenar and soleus muscles (McComas, Sica, Upton, Longmire and Caccia, 1973; Sica, McComas, Upton and Longmire, 1974). The value of the motor unit estimating technique for the diagnosis of patients with various types of peripheral nervous system disorder has been stressed (McComas et al, 1971b; Brown, 1973; Sica et al, 1974) and the technique has shed new light on the behavior of motor units in such conditions as muscular dystrophy, myasthenia gravis and ageing (see, for example, McComas, Sica and Campbell, 1971). During the past 3 years we have had the opportunity to study some patients repeatedly and follow the way in which the numbers and sizes of motor units may alter with the elapse of time. From a total of more than a hundred patients, we now report the results in five. These five were chosen, partly because they were investigated thoroughly, and partly because they all demonstrated, at some time, a recovery of motoneuron function. In two of these the neuropathy had been induced by drugs and, following cessation of therapy, the anticipated recovery took place. The remaining three patients are of more interest. In one there was the rapid reversal of the motoneuron dysfunction which we have come to recognize as a feature 
of thyrotoxicosis (McComas, Sica, McNabb, Goldberg and Upton, 1974). In another patient a striking, though transient, recovery occurred spontaneously in the course of chronic renal failure. The fifth patient had familial motoneuron disease and exhibited remarkable fluctuations in motoneuron function. One facet of this study is that, by comparing the numbers of operative motor units with the functional muscle mass, it has been possible to document the acquisition of muscle fibers by the recovering motoneurons and to look for evidence of remodelling of unit architecture. Lastly, the study has provided additional evidence for the existence, in certain disorders, of non-transmitting synapses (McComas et al, 1974d).

\section{METHODS}

The clinical features of the five patients are described in the Results section. The methods for estimating numbers and sizes of functioning motor units are those of McComas et al (1971b), McComas et al (1973), and Sica et al (1974). In addition, each of the patients underwent a comprehensive electromyographic examination on one or more occasions during the course of their illnesses. The techniques for recording orthodromically-conducted sensory nerve potentials with surface electrodes were those employed by convention (McComas et al, 1974d), as were the measurements of maximum impulse conduction velocity and the examination of muscles with coaxial needle electrodes (Disa type 9013L0501).

\section{RESULTS}

Case 1: Disulfiram neuropathy (Figures 1,2)

Patient K.S. was a 37 year old woman with a 20 year history of chronic alcoholism. On June 28, 1972, treatment was started with disulfiram (Antabuse), $0.5 \mathrm{~g}$ orally each day. Twelve weeks later the patient sought advice for tingling and numbness in the left hand and forearm, blurred vision, and unsteadiness while walking. On exami-

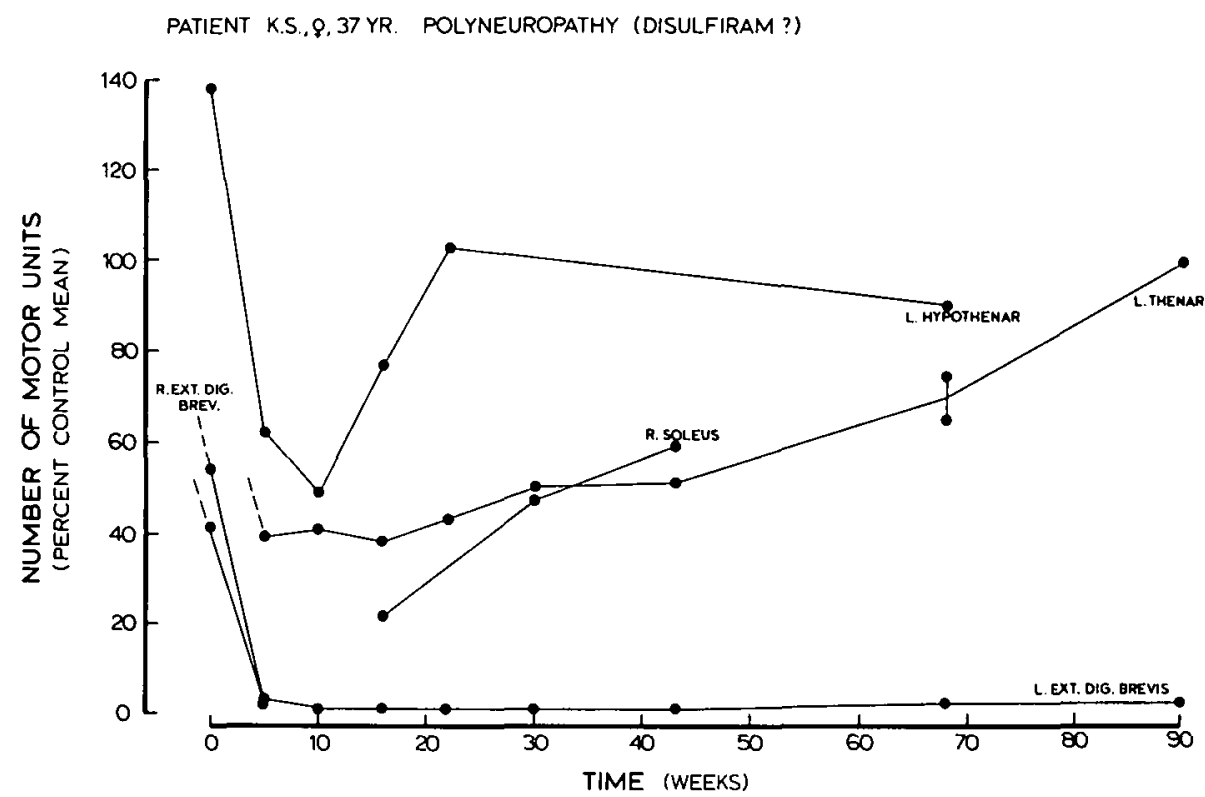

Figure 1. Numbers of functioning motor units in muscles of patient K. S. (Case 1) during the course of a polyneuropathy probably induced by disulfiram (see text). In this figure and in Figs. 3, 4 and 6 the numbers of motor units have been expressed as percentages of the following control mean values ( $\pm S$.D); - extensor digitorum brevis, $208 \pm 63$; thenar group, $340 \pm 87$; hypothenar, $380 \pm 79$; soleus, $846 \pm 193$ (see McComas, Sica and Upton, 1974).

nation no sensory abnormalities were noted except for Rombergism; her ankle jerks were present but diminished. In view of a long and complicated psychiatric history involving numerous transitory symptoms a decision was made to continue the disulfiram under strict clinical surveillance. By November 10 , 1972, 19 weeks after starting treatment, the sensory symptoms had become more pronounced ; a burning sensation and a feeling of numbness were present in both feet. On examination, sensation to light touch and pin-prick was diminished up to the mid-calf level with loss of vibratory sensibility at the ankles; jointposition and temperature sensations appeared to be normal. The ankle jerks could not be obtained, but other tendon reflexes were normal. There was a suggestion of wasting of the calf muscles, but dorsi- and plantar-flexion of the ankles were of normal strength. No abnormality was noted in the arms. A clinical diagnosis of progressive polyneuropathy was made and the disulfiram was stopped. In fact this diagnosis had been made 3 weeks earlier on the basis of a slightly re- duced number of functioning motor units in the left EDB muscle ( 112 units; lower limit of normal, 120 units) and a sural nerve response which was at the lower end of the normal range for amplitude $(5 \mathrm{uV})$.

At the second EMG examination, 5 weeks after the first, it was evident that the functional muscle denervation had progressed (Fig. 1). There were now only 3 motor units remaining in the right EDB and there was a moderate depletion of the left thenar population (141 units; lower limit of normal, 220 units). In contrast, the left hypothenar value was borderline ( 249 units; lower limit of normal, 250 units). It is of interest that the maximum evoked response in the right EDB was only $25 \mathrm{uV}$, which suggested that even in the 3 remaining motor units there had been a loss of muscle fibers. In terms of the neurogenic hypothesis of McComas et al (1971a), this observation indicated that the 3 corresponding motoneurons had entered a dysfunctional ('sick') phase. The left EDB muscle was explored with a coaxial electrode; no volitional activity could be detected even though the maximum evoked response was 
PATIENT K.S., \%.37YR. POLYNEUROPATHY (DISULFIRAM?)

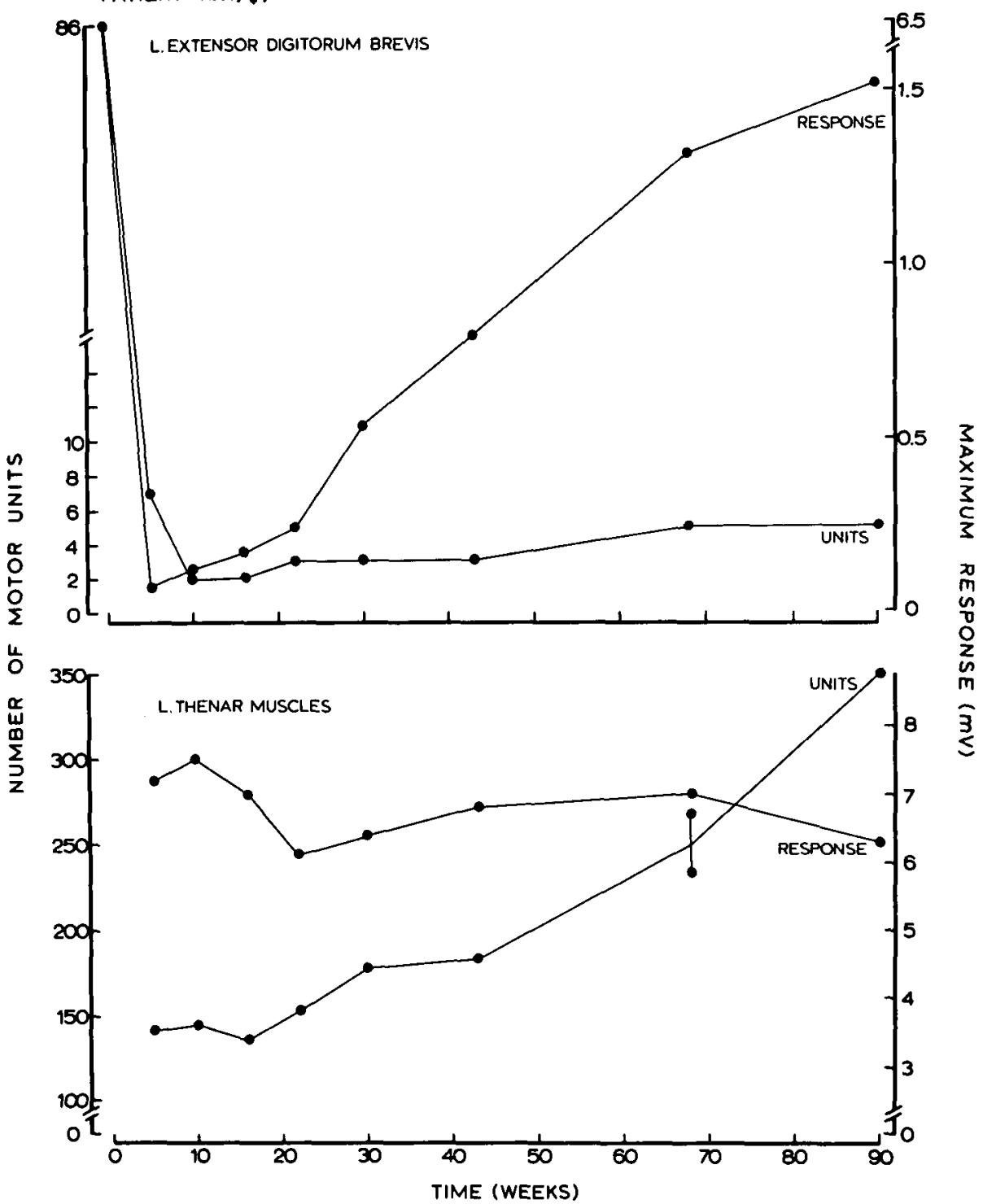

Figure 2. Comparison of the numbers of functioning motor units and maximum evoked muscle responses in the left extensor digitorum brevis (upper section of figure) and left thenar muscles (lower section) of Case 1, at different times in the course of the polyneuropathy (see text).

composed of 7 small increments. These findings could be explained by a failure of impulse propagation in a region of the axons proximal to the ankle or by an inability of the descending facilitatory pathways to excite the surviving motoneurons. The presence of fibrillations and sharp wave activity indicated that many axons had degenerated, rather than merely exhibiting a block of impulse conduction (see McComas, Jorgensen and Upton, 1974). In spite of the severe deterioration in muscle function, the sural nerve responses had not changed significantly (6uV on both sides).

At the third examination (10 weeks; Fig. 1) the left hypothenar and EDB motor unit counts had fallen still further, even though the patient was no longer receiving disulfiram. During the remainder of the period of observation (from 16 to 90 weeks; Fig. 1) functional improvement took place in all the muscles studied, but the rates and extents varied. Fig. 1 shows that, although both the hypothenar and thenar muscles recovered completely, the process was much swifter in the former. In distinction to the hand muscles, the EDB muscle showed very little improvement in the number of functioning motor units, since these had only risen to 5 from a minimum of 2 in the space of 80 weeks. Fig. 2 (upper) shows that the EDB was a more effective muscle, nonetheless, for the maximum evoked response had increased to $1.52 \mathrm{mV}$ from a value of $.075 \mathrm{mV}$, a twenty-fold increase. Fig. 2 (lower) illustrates a second paradox; the undoubted recovery of motor unit function which took place in the thenar muscles was not associated with any significant change in the maximum evoked muscle response. The probable reasons for this contrasting behavior in the thenar and EDB muscles are given in the Discussion.

Case 2: Vincristine neuropathy (Fig. 3)

Patient F.T. was one of 10 patients with vincristine neuropathy studied serially. He was a 35 year old plumber who underwent excision of an embryonal carcinoma of the testis on November 14, 1972; a block dissection of pelvic lymph nodes was also performed. At this time he was given a course of intravenous treatment with vincristine $1.2 \mathrm{mg}$.(once), methotrexate (45 mg. on 2 days), cyclophosphamide (Procytox; $450 \mathrm{mg}$. on 2 days) and 5 -fluorouracil ( $450 \mathrm{mg}$. on 5 days). This course was then repeated twice at 3 weekly intervals. By January 12, 1973, the patient had developed severe numbness and tingling in his hands and feet together with weakness and muscle wasting. Although generalized, the latter was most marked in distal muscles of the limbs.

The initial electrophysiological examination revealed a striking loss of functioning motor units in the thenar and extensor digitorum brevis muscles on both sides (Fig. 3). In contrast, the left hypothenar value was normal (290 units) and the right moderately reduced (93 units). Compound action potentials were diminished in sensory fibers of the radial, median, ulnar and sural 
nerves. The 5-day course of chemotherapy was repeated at 3 weekly intervals until April 24, 1973 (24 weeks; Fig. 3); by this time the patient's weakness had increased, the sensory potentials had undergone further reduction and only 3 functioning motor units remained in the right EDB muscle. In view of the deteriorating neurological condition vincristine was withdrawn, but treatment with the other drugs maintained at 3 weekly intervals. Five weeks later (29 weeks; Fig. 3) the left thenar count was larger, as was the maximum thenar muscle response. At 45 weeks the right EDB count was improving and sensory nerve potentials were larger. At this point the 5-day course of chemotherapy (5-fluorouracil, methotrexate and cyclophosphamide) was given at 6-weekly, rather than at 3-weekly, intervals. At the last occasion of study ( 86 weeks; Fig. 3), the left thenar population was within the normal range (i.e. greater than $63 \%$ of the control mean) and the functional muscle fiber bulk, indicated by the maximum response, had increased to a similar extent. The right EDB population, although still below the lower limit of the normal range, had increased to approximately 27 percent of the control mean from a minimum value of 1 percent (only 3 units were detected at the 24th and 29th weeks).

\section{Case 3: Thyrotoxicosis (Fig. 4).}

K.W. was one of 12 patients with thyrotoxicosis studied serially. She was a 34-year-old woman with a six-month history of increased appetite, sweating, intolerance to warmth, and nervousness. She had not lost weight but she tired easily; she had no muscle stiffness or cramps. On examination she had slight proptosis of both eyes, a fine tremor and a diffusely enlarged thyroid; her pulse rate was $112 /$ minute at rest. All her thyroid function tests were mildly elevated - serum thyroxine 11.2 ug\% (normal, 4-11\%), resin uptake $40 \%$ (normal, 25-35\%) and $I^{131}$ uptake $57 \%$ (normal, 10-45\%). Treatment with radio-iodine ( $3 \mathrm{mci}$ ) was given 4 days
F.T. , $\sigma^{*}, 35$ YR. VINCRISTINE NEUROPATHY

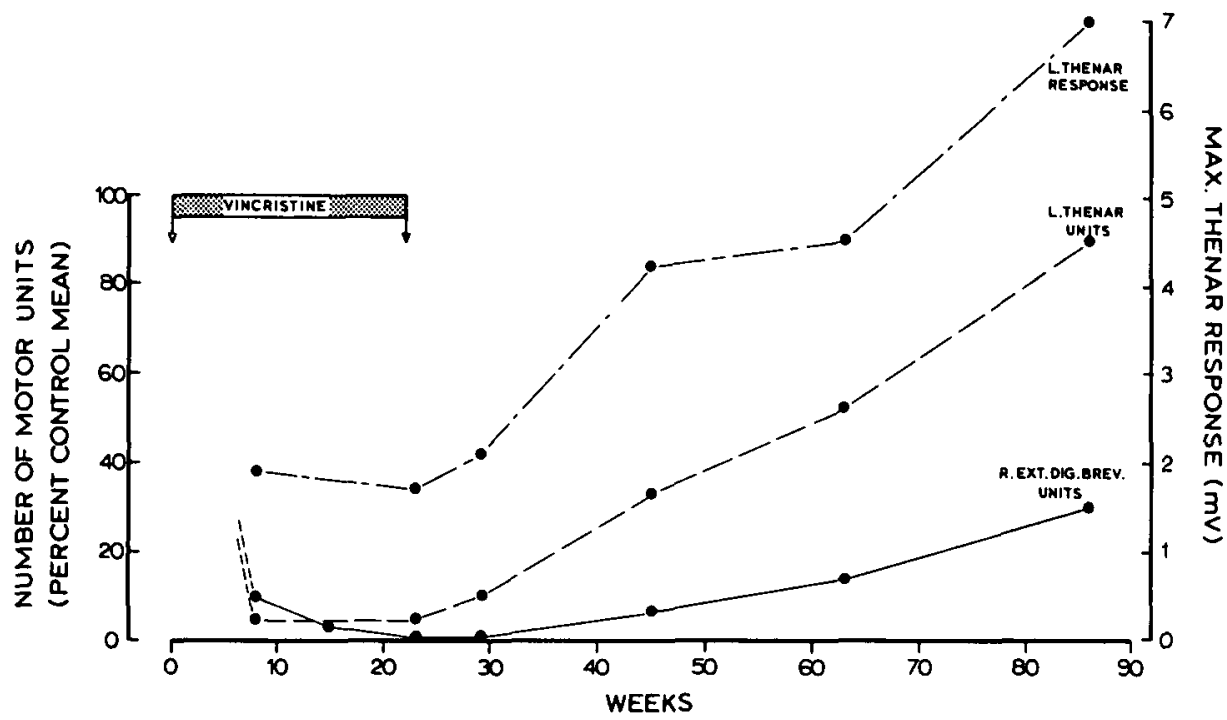

Figure 3. Numbers of functioning motor units in the right extensor digitorum brevis and left thenar muscles of patient $F$. T. (Case 2) during a polyneuropathy induced by vincristine (see text). Maximum evoked thenar responses are also shown. before the first motor unit study; a beta-adrenergic blocking agent was not administered. This study revealed a loss of functioning motor units which was more severe in EDB than in the thenar muscles; the hypothenar muscles were unaffected. When studied 2 weeks later, significant improvements in EDB and thenar muscles had taken place and appeared to become complete in the following 2 week period. Inspection of Fig. 4 shows that the maximum evoked muscle response did not change in the thenar muscles, whereas in EDB it remained proportional to the increasing numbers of motor units.
Figure 4. Numbers of functioning motor units and maximum evoked muscle responses in the left extensor digitorum brevis (left) and left thenar muscles (right) of patient $\mathrm{K}$. $\mathrm{Q}$. (Case 3) during recovery from thyrotoxicosis (see text). Note that, unlike the other figures, this figure expresses the time dimension in days rather than in weeks.

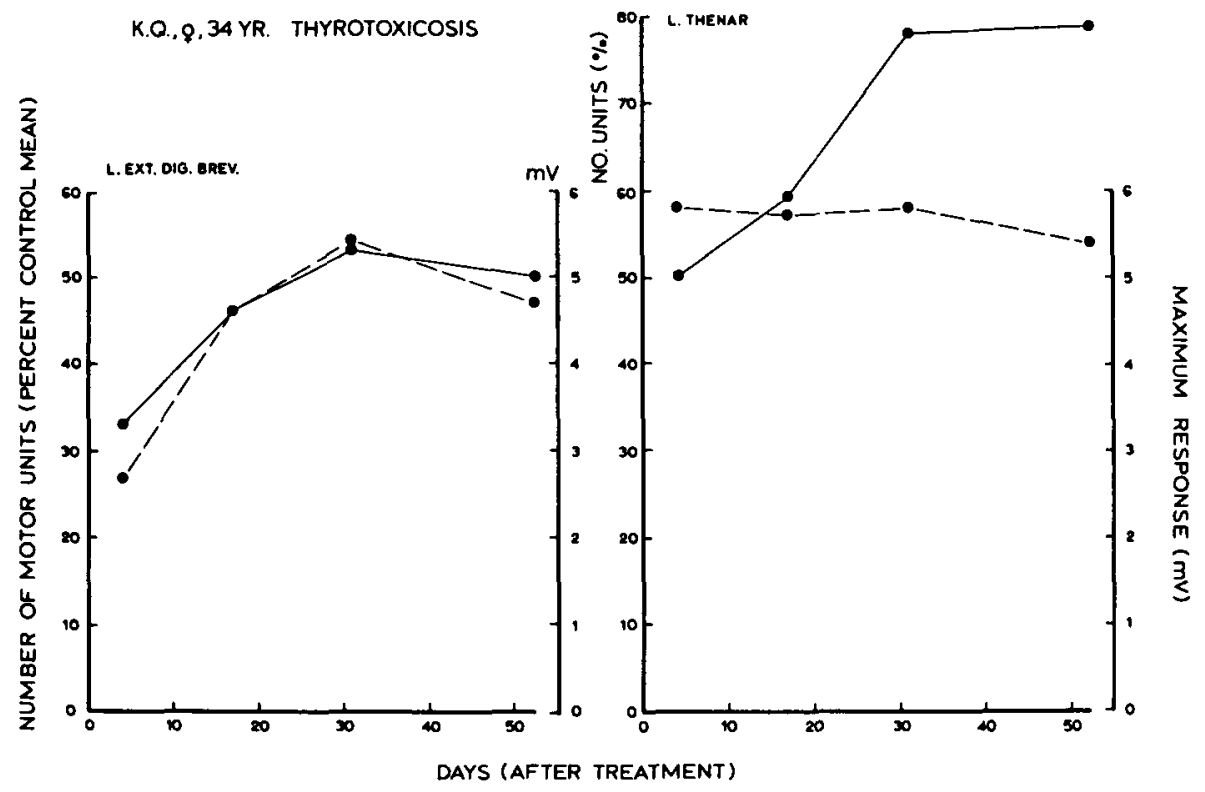




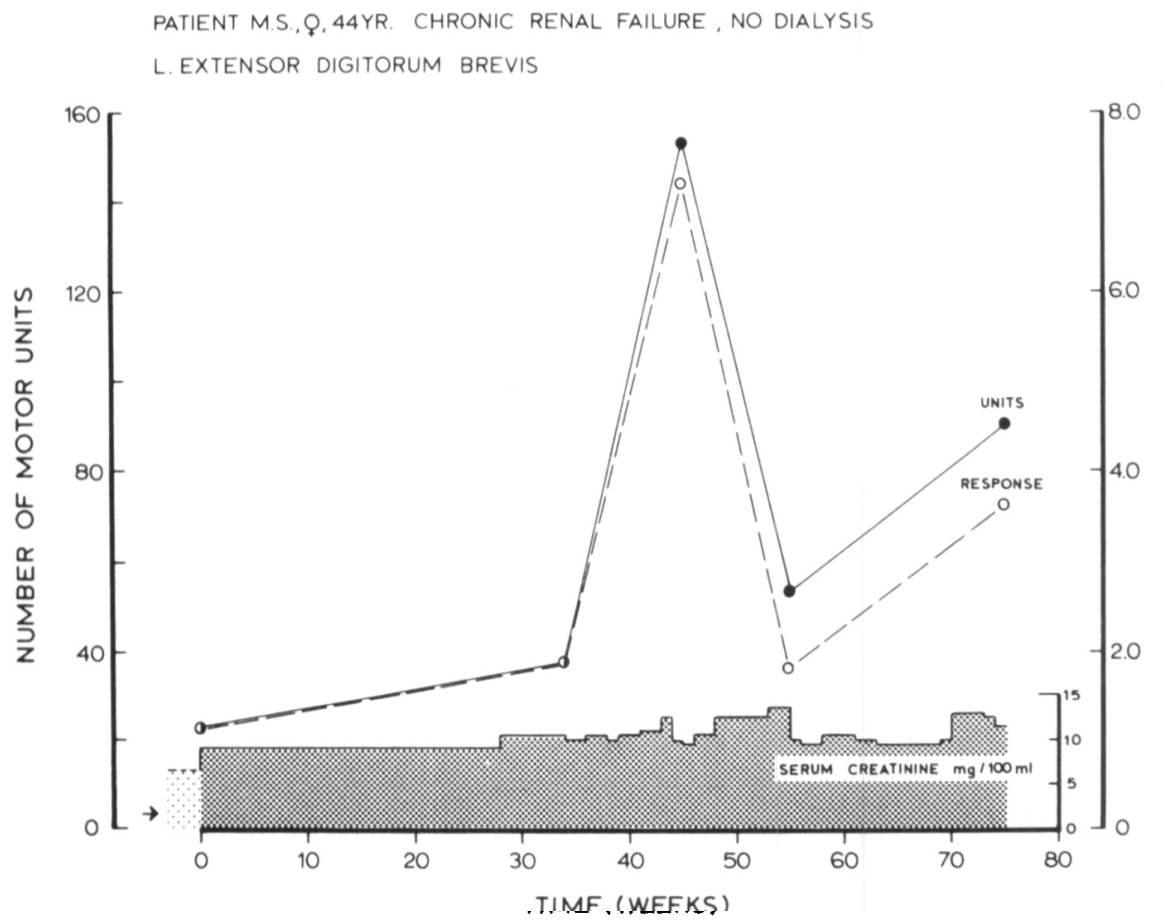

Figure 5. Number of functioning motor units and maximum evoked muscle responses in the left extensor digitorum brevis muscle of patient M. S. (Case 4). At bottom are the serum creatinine levels, which showed no correlation with the determinations of neuromuscular function (see text).

\section{Case 4: Chronic renal failure (Fig.} 5).

Patient M.S. was one of 50 patients with chronic renal failure studied serially. She presented in July 1972, age 44, with symptoms of nausea, vomiting, shortness of breath, angina of effort, swelling of her ankles and hematuria. Her kidneys were enlarged on palpation and were shown radiologically to be polycystic. Her blood pressure, standing, was $150 / 100 \mathrm{~mm}$. $\mathrm{Hg}$; her fundi appeared normal and no abnormalities were noted on examination of her nervous system. Laboratory examination revealed the following serum electrolyte values $(\mathrm{mEq} / \mathrm{l})$ : - sodium, 137; potassium, 5.0: chloride, 107: bicarbonate, 17 . Other biochemical values (in mg. \%) were: - calcium, 8.5; phosphorus, 6.2 ; blood urea nitrogen, 85 ; creatinine, 6.6 . She had a normochronic anemia (hemoglobin, $8.8 \mathrm{~g} \%$ ). At this stage her only medication was Aldomet $250 \mathrm{mg}$., three times daily. Upon electrophysiological examination a severe loss of functioning motor units was noted in the left EDB muscle (Fig. 5): about 23 were left. The motor unit potentials of the surviving functional units were of normal size (mean $46 \mathrm{uV}$ ) and no spontaneous activity could be detected during exploration of the muscle with a coaxial electrode.

In contrast to EDB, the hypothenar motor unit population was normal (417 units). Sensory nerve responses were diminished in the sural nerves but normal elsewhere. At the second EMG examination (34 weeks) the left EDB still showed severe functional denervation while the hypothenar muscle remained normal; a thenar study showed moderate denervation (166 units). The third electrophysiological study was undertaken at 45 weeks; on this occasion the muscle responses were strikingly improved. The EDB motor unit count (153 units) was now within the normal range (above 120 units) and Fig. 5 shows that there had been a proportional increase in the maximum evoked muscle response. In keeping with the EDB results, the thenar muscles also showed a significant improvement with a motor unit count of 286 (normal, above 220) and an enlarged maximum muscle response. Fig. 5 shows that this recovery of muscle function was not associated with any improvement in renal function, for the serum creatinine had risen to 9.9 mg \% while the other biochemical values were not significantly different from those obtained initially. Her clinical condition was slightly better, for she no longer suffered from ankle swelling or chest pain while the nausea and breathlessness were less marked. Ten weeks later, at the time of the next electrophysiological examination, a clear deterioration in muscle function had taken place. Both the motor unit count and the maximum muscle response had fallen in EDB (Fig. 5) and in the thenar muscles. The patient now felt more breathless on exertion and the serum creatinine had risen to $13.4 \mathrm{mg} \%$ and the blood urea nitrogen to $100 \mathrm{mg} \%$; the serum electrolytes were not significantly different. At the next examination ( 75 weeks) there had been some improvement in EDB function, but a further decline in that of thenar muscles while, for the first time, the hypothenar muscles showed evidence of involvement. Once again the serum electrolytes were unchanged while the creatinine had fallen to $11.4 \mathrm{mg} \%$ and the blood urea nitrogen to $60 \mathrm{mg} \%$. Following this visit blood dialysis was started; within six months there had been a return of thenar and hypothenar muscle function to normal, but the left EDB could no longer be tested because of proximity of the arteriovenous fistula.

Case 5: Familial motoneuron disease (Fig. 6).

Patient E.Q. was one of 12 patients with motoneuron disease studied serially. She first presented for electrophysiological study at the age of 37 . For the previous five years she had noticed increasing weakness, muscle fasciculations and tiredness. The weakness was generalized, affecting the trunk and neck as well as the proximal and distal limbs. In spite of this long 


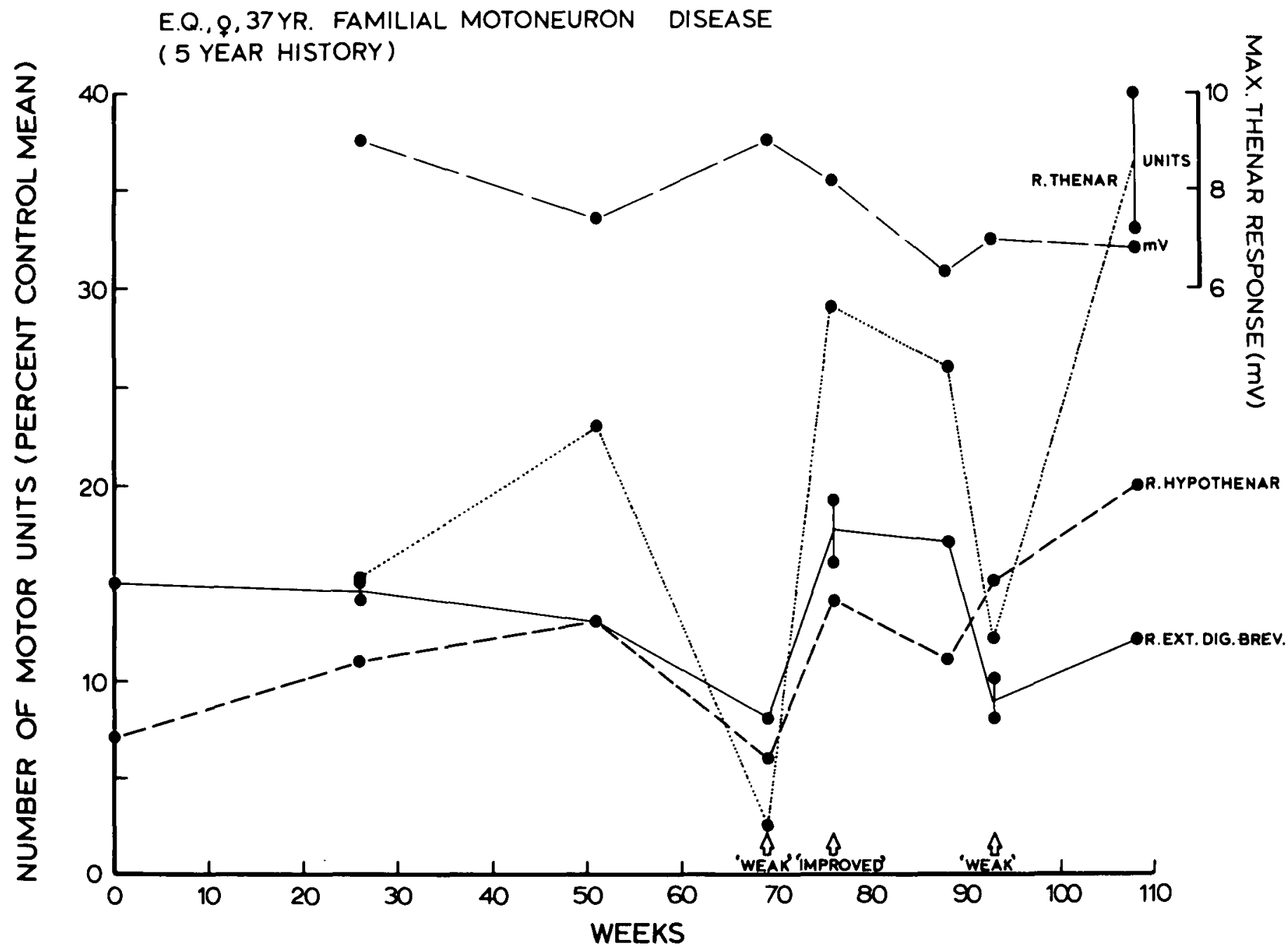

Figure 6. Numbers of functioning motor units in the right extensor digitorum brevis, thenar and hypothenar muscles of patient E. Q. (Case 5) with familial motoneuron disease. Also shown are the responses evoked from the thenar muscles. Arrows at bottom indicate occasions on which the patient volunteered that she felt weaker or stronger than usual.

history she was able to walk reasonably well and to continue her employment as a bank clerk. Upper motoneuron signs were evident. All tendon reflexes were abnormally brisk and Babinski responses were present bilaterally. Her mother had developed a similar disease at the age of 52 and had died after a 5 year illness; she had been diagnosed as having amyotrophic lateral sclerosis (motoneuron disease). Patient E.Q. had been treated at various times with pyridostigmine bromide (Mestinon; Roche), pancreatic extract and Vitamin E; in addition she had received chlordiazepoxide (Librium; Roche) and halperidol (Haldol; McNeil) for an associated depression and anxiety state.
In contrast to the other patients with motoneuron disease studied serially, this patient showed a progression of the disease which was not only much slower but which fluctuated in a remarkable manner. During the first year of observation no significant change had taken place in the hypothenar and EDB motor unit populations though there appeared to be some increase in the thenar population (Fig. 6). At the 69th week the patient complained of increasing weakness and was re-examined. On this occasion all three motor unit populations showed depletion, most marked in the thenar group. Six weeks later the patient was admitted to hospital in order to undergo an empirical trial with corticosteroids.
On admission the patient volunteered that she was feeling much stronger and that she felt treatment would not be necessary. An EMG (76 weeks) showed that all three populations of functioning motor units were larger, the thenar increase being the most striking. This improvement was maintained at the time of the next EMG (at 88 weeks). On the next occasion, when the patient stated that she had again become weaker, two of the motor unit counts were depressed. At the last examination, at 108 weeks, all three motor unit counts had improved, the thenar value being higher than on any of the previous 6 occasions. At this time the patient was unaware of any improvement in strength, but 
her self-assessment may have been influenced by a profound depression. In spite of the extremely marked fluctuations in the number of thenar motor units, the size of the maximum evoked muscle response showed no significant change (Fig. 6).

The EDB and hypothenar responses were also close to their respective original values at the end of the two year observation period; in contrast to the thenar response, both showed a significant diminution at the time of greatest loss of functioning motor units (69 weeks; not shown in Fig. 6).

\section{DISCUSSION}

Studies such as these, with serial estimates of functioning motor units, provide information concerning the behavior of motoneurons in a number of clinical disorders.

\section{Differential vulnerabilities of moto- neurons.}

The first point is that cases 1-4 demonstrate that motoneuron pools differ in their susceptibilities to a variety of noxious agents - vincristine, disulfiram, and thyrotoxic and renal 'factors'. In each of these patients the extensor digitorum brevis (EDB) neurons were affected to a greater extent than the thenar cells, while the hypothenar neurons were least involved. Some motoneurons are consistently more vulnerable than others. This observation has been noted before in relation to ageing (Sica et al, 1974), muscular dystrophy (McComas, Sica, Upton and Petito, 1974) and thyrotoxicosis (McComas et al, 1974d) and there is no satisfactory explanation. It is obvious from a consideration of the thenar and hypothenar muscles that neither axonal length nor motor unit sizes can be the deciding factors since these must be very similar in the two instances. Elsewhere it had been suggested that genetic factors may be involved in determining motoneuron vulnerability (McComas et al, 1973; Fig. 7, b,d). An alternative explanation is that the results are most severe for the EDB and thenar muscles because the de-
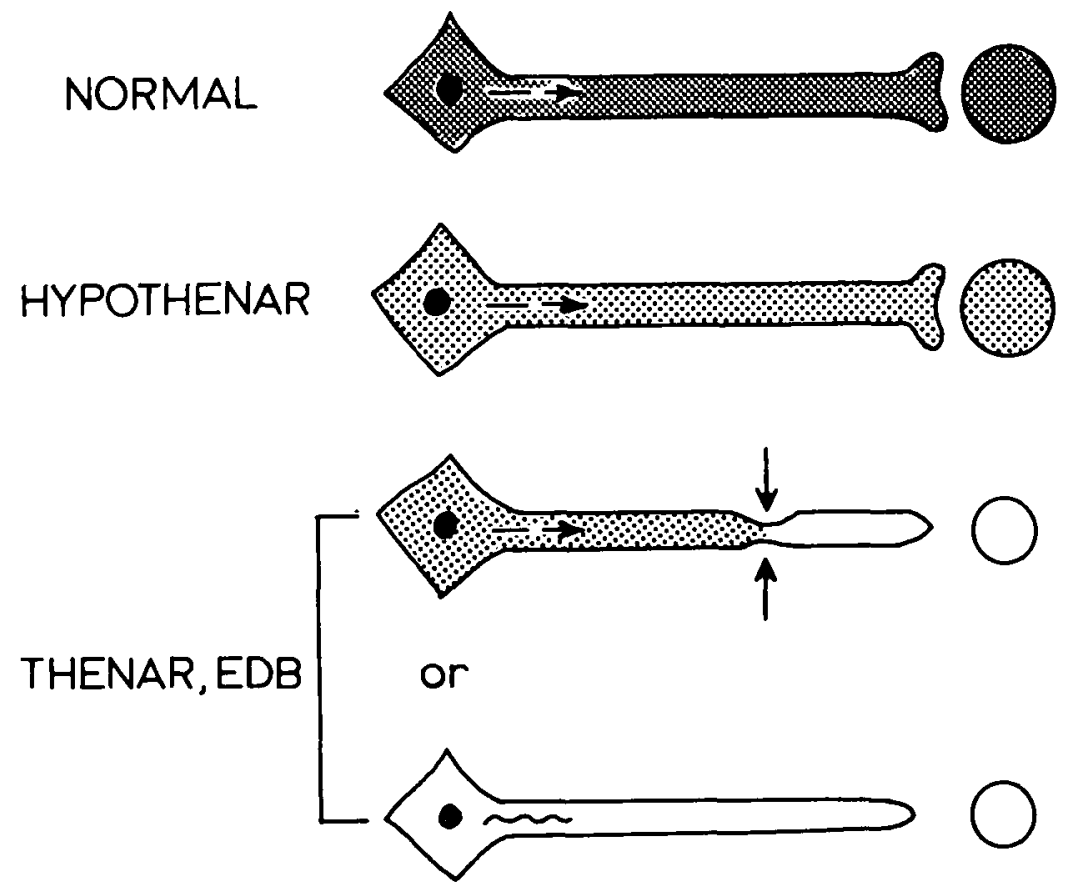

Figure 7. Possible mechanisms underlying differential involvement of motoneuron pools by systemic neurotoxic factors, (a) represents normal situation in which healthy neurons maintain axoplasmic transport of trophic material necessary for maintenance of axon and muscle fiber.

First possibility: Both in (b) and (c) the motoneurons have become dysfunctional ('sick'). Whereas axoplasmic transport from hypothenar motoneurons is still adequate for target structures, that from thenar and EDB neurons is further reduced by compressions of corresponding axons (e.g. in roots, in carpal tunnel, around head of fibula etc.). These compressions are considered to be normally present but asymptomatic.

Second possibility: Due to unspecified genetic factors the hypothenar motoneurons (b) are less affected than the thenar and EDB neurons (d), even though the neurotoxic challenge is the same.

livery of axoplasmically-conveyed trophic material to these muscles is reduced by the double effect of (a) sick (dysfunctional) motoneurons unable to synthesize normal amounts of trophic material and (b) minor degrees of nerve compression, reducing axoplasmic flow (Fig. 7, c; see also Upton and McComas, 1973). Thus, the peroneal nerve to EDB may be compressed under the long extensor tendons or by tightfitting shoes (Jennekens, Tomlinson and Walton, 1972) while the median nerve is predisposed to trauma within the carpal tunnel. The fact that none of our four patients had other EMG evidence of a carpal tunnel syndrome would not necessarily exclude the possibility of minor trauma, although it does make this explanation rather less attractive.
Time course of motoneuron dysfunction.

The present results illustrate another principle of motoneuron dysfunction, namely, recovery begins sooner and proceeds faster in the least affected motoneuron pools. For example, in Case 1, function in EDB muscles had just started to improve at a time when recovery in the hypothenar group was complete and in the thenar muscles was far advanced (Fig. 1). Similarly, in Case 2 , the thenar motor unit population had returned to normal while more than half the EDB pool was still dysfunctional.

However, the results show that even in the most severely affected motoneuron pools it is often possible for recovery to take place provided sufficient time is allowed to elapse 
after the noxious agent has been withdrawn. As an illustration, only 3 motor units were operational at one stage during the course of the vincristine-induced neuropathy in Case 2; yet at the time of the most recent observation ( 86 weeks later) about 57 motoneurons had recovered function. Not all motoneurons recover; in Case 1 the EDB count at 90 weeks had only increased to 5 from a minimum of two.

In summary, and setting aside this last result, the present study demonstrates that motoneurons combine an extreme sensitivity to the presence of adverse environmental factors with a remarkable resilience during subsequent periods of normality.

Mechanisms of motoneuron dysfunction and recovery.

It is customary to differentiate two basic and apparently different types of peripheral nerve disorder. One of these is segmental demyelination which until recently has been thought to reflect a primary disorder of the Schwann cell. Dyck, Johnson, Lambert and O'Brien (1971) have now suggested that in uremia the non-random distribution of the demyelination may signify an underlying neuronal disorder. The other type of nerve lesion is the 'axonal' or 'dying-back' type (Cavanagh, 1964). In this variety a lesion of the neuron soma is considered to interfere with axoplasmic flow and thereby to produce degeneration of the axon, commencing in its most distal regions, that is, at the neuromuscular junction. Following motor unit studies in a variety of diseases, McComas et al (1974c) suggested that the dying-back process might be subdivided into seven stages, depending on the numbers of neuromuscular junctions involved and the severity of the associated synaptic dysfunction at each. The scheme was imprecise because the relative importance of axoplasmic flow and of excitation in maintaining neurotrophic control of the muscle fiber was unknown. In man, at least, the situation has become clearer from a recent study of neurapraxic lesions (McComas et al, 1974a). It

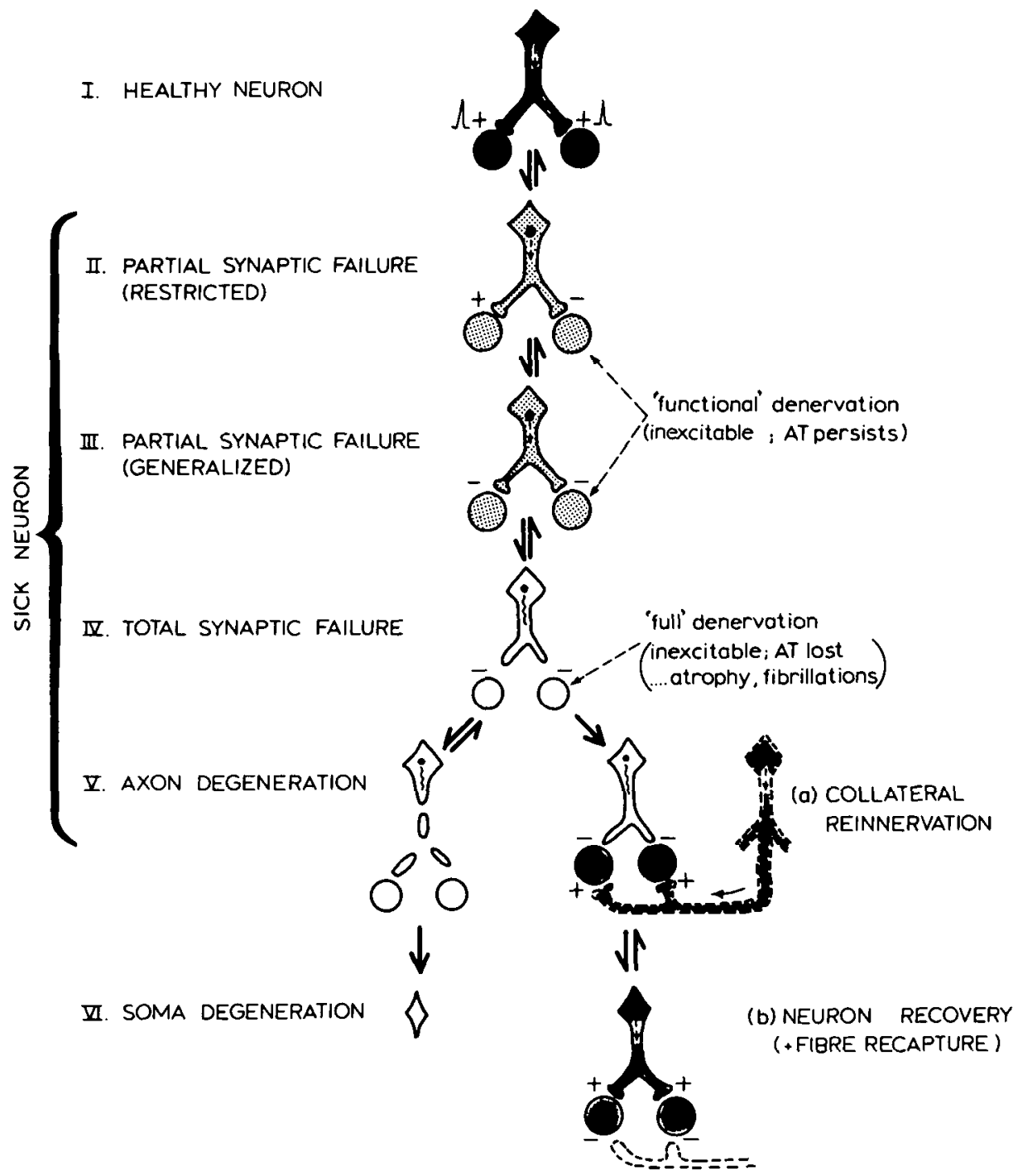

Figure 8. Stages I to VI depict increasing motoneuron dysfunction, culminating in irreversible degeneration of the neuron soma. The presence of trophic material conveyed by axoplasmic transport (AT) is indicated by the arrows directed from the cell soma, while the amount of material is represented by the intensity of the stippling. Excitable and non-excitable synapses are indicated by positive and negative signs respectively. This 6-stage scheme supplants the 7-stage one put forward by McComas et al (1974c), but is compatible with the detailed series of events postulated by McComas et al (1974a) to occur following axonal crush. At lower right are shown two other mechanisms involved in the restoration of muscle function, collateral reinnervation (a) and neuron recovery with recapture of muscle fibers (b). See text for full description.

now appears that muscle fibers no longer receiving impulses from their parent motoneurons may fail to exhibit other clinical and electrophysiological features of denervation. The implication is that the denervation phenomena have been prevented by axoplasmic flow; such fibers may therefore be considered as 'functionally' rather than 'fully' denervated. This concept of functional denervation has proved of great value in an understanding of the changes observed in the present study. It has also enabled the graded motoneuron dysfunction scheme of McComas et al (1974c) to be revised (see below).

\section{(a) Functional denervation: partial synaptic failure.}

In the present study functionally denervated fibers have been observed following a systemic lesion. 
The best example of this behavior is the EDB muscle of Case 4, the patient with chronic renal failure. On four occasions reduced numbers of operative motor units were observed in this muscle with concomitant reductions in the sizes of the maximum evoked muscle potentials (Fig. 5). Nevertheless, there were four reasons for postulating that the non-operational synapses still retained connections with the muscle fibers and, although inexcitable, continued to mediate neurotrophic actions. First, the muscle was not atrophied on clinical examination. Second, careful examination of the EDB muscle with a coaxial electrode on these four occasions did not reveal any spontaneous electrical activity characteristic of 'denervation' (i.e. fibrillations and positive sharp waves). Third, the incremental potentials evoked in the surviving motor units were not enlarged: this suggested that the non-functioning muscle fibers had not been available for collateral reinnervation by 'healthy' neurons (though it could be argued that the other neurons were incapable of undertaking collateral innervation because of their dysfunctional state; see McComas et al, 1971a). However, the most convincing evidence favoring the existence of inactive synapses was the rapid recovery of function which took place at the third study. The compact configuration of the maximum evoked response and the normal maximal conduction velocity in motor axons of the peroneal nerve both suggested that recovery of function had not involved regeneration or remyelination of axons. That this transient improvement in function was real, rather than due to experimental error, was indicated by its simultaneous occurrence in the thenar and hypothenar muscles.
Another, but less dramatic, example of reversible functional denervation is the EDB muscle of the thyrotoxic patient K.Q. (Case 3) in which the numbers of motor units and the maximum muscle responses increased proportionately following treatment with I'131 (Fig. 4). Recently McComas et al (1974a) have reported partial synaptic failure following cervical root injury; in that patient it was supposed that the mechanical damage to the axon had interfered with axoplasmic flow and produced 'dying back' effects.

In Fig. 8 the process of functional denervation due to partial synaptic failure has been represented by stages II and III. In stage II only a fraction of the neuromuscular junctions in the individual motor units are affected, causing the maximum evoked muscle response to decrease without a fall in motor unit number, as shown in Fig. 9. This behavior

Figure 9. The electrophysiological correlates of the 6-stage scheme of motoneuron dysfunction (above) are shown. Although stages IV to VI cannot be distinguished from each other, stages I to IV are readily differentiated by determination of the maximum evoked muscle response, the number of functioning units and the presence or absence of spontaneous muscle fiber activity. The figure also shows the findings which characterize collateral reinnervation and motoneuron recovery with fiber recapture (see text and Fig. 8).

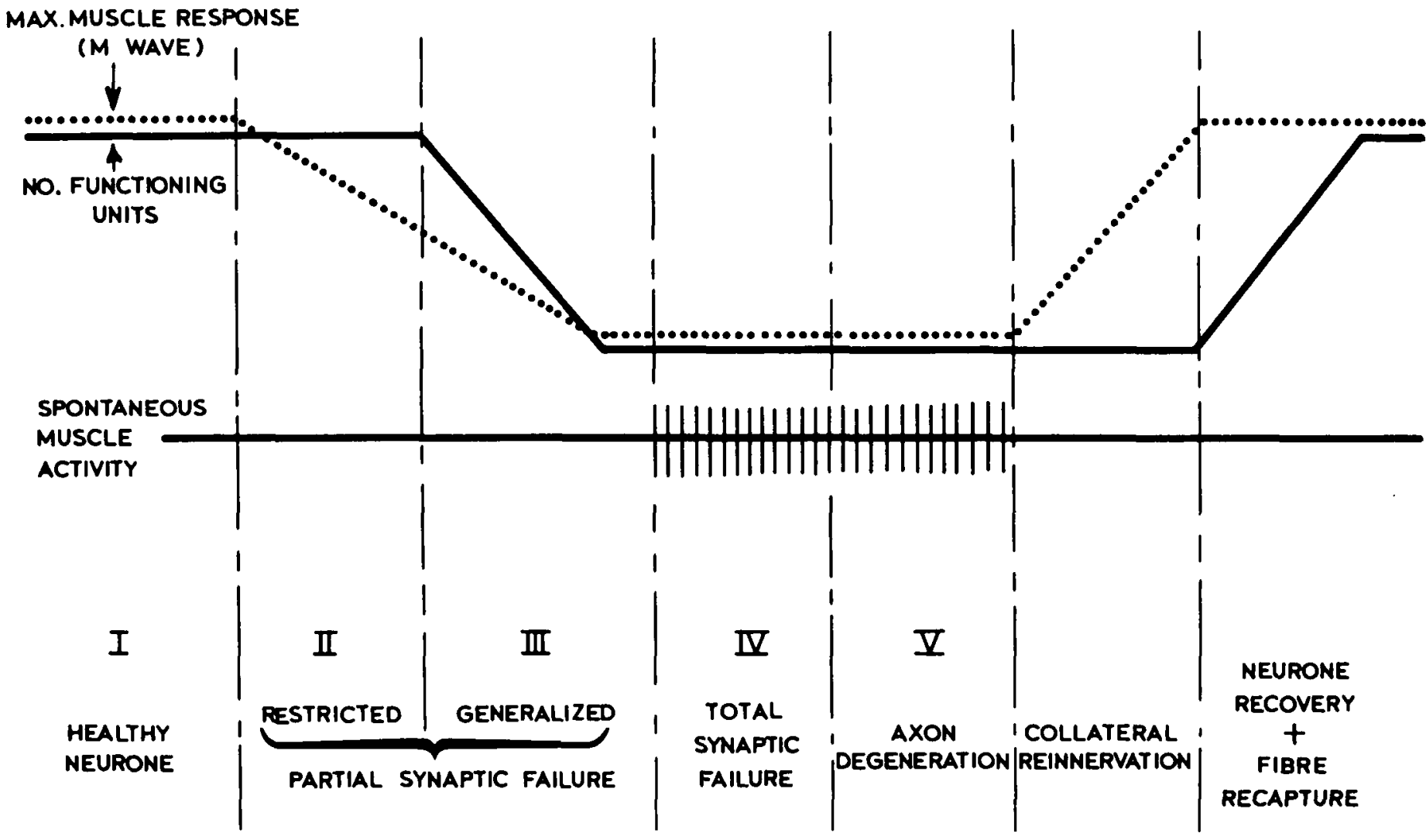


has sometimes been observed and is the earliest detectable stage of motoneuron dysfunction. In stage III all junctions are affected and the fall in maximum evoked muscle matches the loss of functioning motor units (see Fig. 9). As the EDB muscles of Cases 1 and 4 illustrate, stages II and III can readily be reversed by reactivation of the dormant synapses.

\section{(b) Full denervation: total synaptic} failure.

In some muscles of the present study the presence of inexcitable muscle fibers was associated with muscle atrophy and fibrillations during the active phase of the illness; the severely affected EDB muscles of Cases 1 and 2 were the most florid examples. The absence of any detectable neurotrophic action on the muscle fibers, in contrast to the fibers considered in the previous section, suggested that synaptic failure was now complete and that axoplasmic flow had been abolished as well as neuromuscular excitation (stage IV in Fig. 8; see also Fig. 9). It is obviously possible that in such muscles the neuromuscular junctions had degenerated and in some instances, distal regions of the axons too (Fig. 8, stage V), but we have no morphological data on our patients to substantiate this reasoning.

In the recovery from total synaptic failure the maximum evoked muscle potential should increase with the number of functioning motor units and this was observed in the EDB muscle of Case 2 (vincristine neuropathy). In theory it is possible that the newly formed synapses may pass through an intermediate phase of partial synaptic failure in which the atrophy and fibrillations disappear with little increase in motor unit number or in maximum evoked muscle response. To have explored this possibility would have necessitated repeated examinations with coaxial electrodes and these were not performed. However, the predicted behavior has been noted in two other patients studied after nerve injury (unpublished observations). It is of interest that, in the frog, axons regenerating after a nerve crush may also enter a phase in which the newly established synapses are non-transmitting, but already active in a trophic capacity (Miledi, 1960).

\section{(c) Collateral reinnervation.}

Once a muscle fiber is fully denervated it becomes available for collateral reinnervation by sprouts of surviving functional motor axons (Fig. 8, a). This process has been documented experimentally by Kugelberg, Edstrom and Abbruzzese (1972) and electrophysiological evidence for its occurrence in chronic human neuropathies has been reported previously by McComas, Sica, Campbell and Upton (1971). Additional observations have been made in the present study, the best example being the EDB muscle of patient K.S. (Case 1; disulfiram toxicity; Fig. 2, upper). During a phase when only 3 motor units remained functional the maximum muscle response grew from $250 \mathrm{uV}$ to $800 \mathrm{uV}$ over the space of 21 weeks. More impressive was the observation that the maximum muscle response had increased from .075 $\mathrm{mV}$ at 5 weeks to $1.52 \mathrm{mV}$ at 90 weeks (a twenty-fold increase) even though the number of functioning units had declined from 7 to 5 (see also Fig. 9).

\section{(d) Recapture of annexed muscle} fibers.

In some muscles in which collateral reinnervation had taken place it appeared that muscle fibers might be surrendered by the adoptive motoneurons to the newly recovered original neuron (Fig. 8b) or shared by the two neurons. One example was the thenar muscle group of patient K.Q. (Case 3; thyrotoxicosis) in which the number of functioning motor units increased from approximately 170 to 270 following treatment (Fig. 4). The fact that the maximum evoked muscle response did not increase suggests that the muscle fibers had been redistributed or else shared (see Fig. 9). Similar observations on thyrotoxic patients have been made previously (McComas et al, 1974d), but in the present study this remodelling of units was also shown to take place in the recovery from disulfiram toxicity (thenar muscle of Case 1; Fig. 2, lower). In this last example the maximum muscle response fluctuated between 6.1 and $7.5 \mathrm{mV}$, an insignificant variation, while the motor unit population steadily increased from 136 to 350 (see also Fig. 9). A more remarkable instance of this type of neuromuscular behavior is the patient with motoneuron disease, E.Q. This patient, a 37 year old lady with a familial form of the disease, was studied repeatedly over a two year period. Although each of the muscles investigated had evidence of severe chronic denervation, the numbers of functioning motor units were seen to fluctuate to an extent which was beyond the limits of experimental error and consistent among the muscles studied (Fig. 6). In spite of the changes in the numbers of functioning units, the corresponding maximum evoked muscle responses were little altered, as exemplified by the thenar results in Fig. 6. It was of added interest that the patient's voluntarily expressed feelings of strength or weakness correlated directly with the numbers of functioning units. Although transient remissions of neuronal function have not been observed in 11 other patients with motoneuron disease studied serially, it has recently been noted in a 17 year old boy with peroneal muscular dystrophy (unpublished observations).

The interpretation of these results, as stated, is that the 'original' motoneurons are able to reactivate or else regenerate their synapses and thereby recapture muscle fibers from adoptive motoneurons. It follows that, at a certain phase of the disorder, each of the affected muscle fibers must have had two synapses, one from each neuron (Fig 8b). In the patient with motoneuron disease the adoptive synapses probably persisted in order to account for the observed changes during periods of neuronal relapse. In the thenar muscles of patients K.Q. (Case 4; thyrotoxicosis) and K.S. (Case 1; disulfiram-induced neuropathy) the evidence available suggests that functional connections were proba- 
bly relinquished. If this were not so, excessively large motor unit potential increments should have been encountered during sampling and the estimated numbers of motor units, although larger, should not have returned to pre-morbid values. These findings are at variance with those in the rat. Tonge (1974) found that, during recovery from a nerve crush, the original motoneurons would form synapses which usually remained inactive unless the axons of the adoptive motoneurons were interfered with. It is possible that this discrepancy between the two studies could reflect species differences (man and rat). An alternative explanation lies in the contrasting types of nerve injury. In Tonge's experiments nerve crush was used whereas in our study systemic toxic factors were involved.

In conclusion, the present investigation has been concerned with the ways in which motoneurons lose and regain their function under the influence of a variety of external stimuli. It has been shown that the steps in motoneuron dysfunction and recovery can be distinguished by three simple electrophysiological criteria, (i) the size of the maximum evoked muscle response, (ii) the number of functioning motor units, and (iii) the presence or absence of spontaneous muscle fiber activity (Fig. 9). Although detailed knowledge of the mechanisms underlying these steps must inevitably await experimentation on appropriate animal models, there are now good reasons for be- lieving that the repertoire of disordered neuronal bahavior is more complex than had previously been thought.

\section{REFERENCES}

BROWN, W. F. (1972). A method for estimating the number of motor units in thenar muscles and the changes in motor unit count with ageing. Journal of Neurology, Neurosurgery and Psychiatry, 35, 845-852.

BROWN, W. F. (1973). Thenar motor unit count estimates in the carpal tunnel syndrome. Journal of Neurology, Neurosurgery and Psychiatry, 36, 194-198.

CAVANAGH, J. B. (1964). Peripheral nerve changes in orthocresyl phosphate poisoning in the cat. Journal of Pathology and Bacteriology, 87, 365-383.

DYCK, P. J., JOHNSON, W. J., LAMBERT, E. H. and O'BRIEN, P. C. (1971). Segmental demyelination secondary to axonal degeneration in uremic neuropathy. Mayo Clinic Proceedings, 46, 400-431.

JENNEKENS, F. G. I., TOMLINSON, B. E. and WALTON, J. N. (1972). The extensor digitorum brevis: histological and histochemical aspects. Journal of Neurology, Neurosurgery and Psychiatry, 35, 124-132.

KUGELBERG, E., EDSTROM, L. and ABBRUZZESE, M. (1970). Mapping of motor unit in experimentally reinnervated rat muscle. Interpretation of histochemical and atrophic fiber patterns in neurogenic lesions. Journal of Neurology, Neurosurgery and Psychiatry, 33, 319-329.

McCOMAS, A. J., JORGENSEN, P. B. and UPTON, A. R. M. (1974a). The neurapraxic lesion. A clinical contribution to the study of trophic mechanisms. Canadian Journal of Neurological Sciences. 1, 170-179.

McCOMAS, A. J., SICA, R. E. P. and CAMPBELL, M. J. (1971a). 'Sick' motoneurons. A unifying concept of muscle disease. Lancet, 1, 321-325.

McCOMAS, A. J., SICA, R. E. P. and UPTON, A. R. M. (1974b). Multiple muscle analysis of motor units in muscular dystrophy. Archives of Neurology, 30, 249-251.

McCOMAS, A. J., FAWCETT. R. R. W., CAMPBELL, M. J. and SICA, R. E. P. (1971b). Elect rophysiological estimation of the number of motor units within a human muscle. Journal of Neurology, Neurosurgery and Psychiatry, 34, 121-131.

MCCOMAS, A. J., SICA, R. E. P., CAMP$B E L L, M$. J. and UPTON, A. R. M. (1971c). Functional compensation in partially denervated muscles. Journal of Neurology, Neurosurgery and Psychiatry, 34, 453-460.

McCOMAS, A. J., SICA, R. E. P., UPTON, A. R. M. and PETITO, F. (1974c). Sick motoneurons and muscle disease. In Trophic Function of the Neuron (Symposium), ed. Drachman, D. B. Annals of the New York Academy of Sciences, 228, 261-279.

McCOMAS, A. J., SICA, R. E. P., McNABB, A. R., GOLDBERG, W. M. and UPTON, A. R. M. (1974d). Evidence for reversible motoneuron dysfunction in thyrotoxicosis. Journal of Neurology, Neurosurgery and Psychiatry, 37, 548-558.

MCCOMAS, A. M., SICA, R. E. P., UPTON, A. R. M., LONGMIRE, D. and CACCIA, M. R. (1973). Physiological estimates of the numbers and sizes of motor units in man. In Control of Posture and Locomotion (Symposium), ed. Stein, R. B. et al. New York: Plenum Press.

MILEDI, R. (1960). Properties of regenerating neuromuscular synapses in the frog. Journal of Physiology, 154, 190-205.

SICA, R. E. P., McCOMAS, A. J., UPTON, A. R. M. and LONGMIRE, D. (1974). Motor unit estimations in small muscles of the hand. Journal of Neurology, Neurosurgery and Psychiatry, 37, 55-67.

TONGE, D. A. (1974). Synaptic function in experimental dually innervated muscle in the mouse. Journal of Physiology (Proceedings). In press.

UPTON, A. R. M. and McCOMAS, A. J. (1973). The double crush hypothesis in nerve entrapment syndromes. Lancet, ii, 359-362. 